EPiC Series in Language and Linguistics
Volume 3, 2018, Pages 79-84
LSP in Multi-disciplinary contexts of
Teaching and Research. Papers from the
16th International AELFE Conference

\title{
El lenguaje para fines artísticos: las traducciones de tondo al español
}

\author{
Elena Carpi \\ Dipartimento di Scienze Politiche, Università di Pisa, \\ 56124 Pisa, Italy; elena.carpi@unipi.it
}

\begin{abstract}
Este trabajo estudia las traducciones españolas de la palabra italiana tondo, que entra en el español como préstamo del italiano en el lenguaje de la arquitectura. Sin embargo, en italiano tondo se usa también en el lenguaje de la pintura, como testimonia la famosa obra de Miguel Ángel Tondo Doni.

El estudio del término tondo ejemplifica la metodología utilizada en el marco del proyecto interuniversitario Lessico dei Beni Culturali (LBC) del Dipartimento di Lingue, Letterature e Studi Multiculturali de la Universidad de Florencia, cuya finalidad es la creación de diccionarios monolingües y bilingües relacionados entre sí mediante enlaces hipertextuales, que ayuden a los traductores que se enfrentan a un texto de temática artística a comprenderlo y a escoger los términos adecuados en la lengua meta, con relación al contexto en que aparecen y a las variadas tipologías de discurso de las guías turísticas, de las páginas web de museos o de los catálogos de exposiciones. Para cada palabra estudiada se redacta una ficha-fuente en italiano y otra para cada lengua del proyecto (alemán, inglés, chino, español, francés, portugués, ruso) en perspectiva diacrónica y sincrónica, en la base de textos especializados y diccionarios; las dos perspectivas son necesarias para comprender los conceptos subyacentes a las denominaciones en la lengua fuente, generalmente caracterizadas por un alto grado de variación semántica.
\end{abstract}

Keywords: lenguaje para fines artísticos; lexicología; terminología; traducción 


\section{Introducción}

El lenguaje para fines artísticos (LFA) es quizá uno de los lenguajes especializados menos analizados en el marco de los LSP, a pesar de la importancia que el Patrimonio histórico artístico reviste hoy en día y de la variedad de subconjuntos que integran su discurso. Dicha escasez de estudios léxicos y terminológicos puede acarrear problemas acusados para traductores no especialistas del sector, a la hora de enfrentarse a un texto de temática artística. En efecto, para establecer la correspondencia correcta entre los términos de la lengua fuente y de la lengua meta es necesario tener en cuenta las diversas tipologías de discurso artístico, su variación diacrónica -sobre todo por lo que se refiere a las técnicas y a los materiales (Carpi 2015; Farina 2015)- y los casos de homonimia (Cabré 1993: 218), puesto que en ámbitos diferentes una palabra puede designar conceptos y objetos distintos.

La palabra italiana tondo usada a la vez en el marco de la arquitectura y de la pintura, representa un ejemplo de esta última tipología léxica. Los dos significados se pueden encontrar ya bien distintos en Vasari, que habla de tondo in fresco, tondo di pittura y tondo di marmo:

Sono di mano di Bartolomeo sotto la volta del palagio del podestà alcuni tondi in fresco (Vasari, 1568: 4- 495)

Et ancora in questo tempo abbozzò e non finì due tondi di marmo, uno a Taddeo Taddei, oggi in casa sua, et a Bartolomeo Pitti ne cominciò uno altro [...]. (Vasari, 1568: 6-21)

Venne volontà ad Agnolo Doni, cittadino fiorentino amico suo... d'avere alcuna cosa di Michelagnolo; per che gli cominciò un tondo di pittura, dentrovi una Nostra Donna, la quale inginocchiata con amendua le gambe, ha in sulle braccia un putto e porgelo a Giuseppo, che lo riceve [...]. (Vasari, 1568: 6-22).

El objetivo del presente estudio es mostrar mediante un ejemplo concreto -el análisis de las traducciones españolas del sustantivo italiano tondo- la metodología de trabajo desarrollada en el marco del proyecto interuniversitario Lessico dei Beni Culturali (LBC) del Dipartimento di Lingue, Letterature e Studi Multiculturali de la Universidad de Florencia. El proyecto tiene como objetivo la creación de diccionarios monolingües y bilingües relacionados entre sí mediante enlaces hipertextuales, que ofrezcan a los traductores y a los guías turísticos una herramienta que abarque todos los significados de un término y proponga un equivalente correcto, en una óptica diacrónica y sincrónica. Para la redacción de los diccionarios se adopta la llamada teoría funcional de la lexicografía, que define la función como: «la asistencia que presta una obra lexicográfica para satisfacer los tipos específicos de necesidades de información puntual que pueda tener un tipo específico de posible usuario en un tipo específico de situación extra lexicográfica» (Tarp 2015: 36); la misma línea teórica hace hincapié en la necesidad de que los diccionarios sean digitales y multidisciplinarios.

En la fase preliminar del proyecto se creó una base de datos multilingüe y paralela de textos de temática artística (siglos XVI-XX) pertenecientes a distintos géneros, que se analizaron mediante el programa de concordancias Sketch Engine; este trabajo permitió establecer un listado de términos, que formaron la base del diccionario. Posteriormente, se redactaron las fichas terminológicas de las palabras italianas, con especial atención a la posible variación diacrónica de los términos, mediante la búsqueda en repertorios lexicográficos monolingües, obras especializadas, bases de datos y páginas web de museos; los resultados fueron validados por 
especialistas del sector. Se elaboró una ficha por cada significado de los términos, incluyendo siempre citas tomadas de la base de datos.

Si de la palabra estudiada proceden sintagmas u otras voces creadas por derivación o composición, en el diccionario en línea tendrán un enlace con la palabra base. Con el término tondo se crean a tuttotondo y mezzotondo, cuya traducción al español no se presenta aquí debido a la falta de espacio, que van a ser objeto de un trabajo sucesivo.

Finalmente, para cada lengua del proyecto -en este caso el español- cada grupo de investigadores reproduce la labor del equipo que se ha ocupado de la ficha italiana, añadiendo también el estudio de los diccionarios bilingües de referencia.

\section{Análisis y resultados}

Aplicando la metodología descrita en 1., para el diccionario monolingüe italiano se realizaron dos fichas de tondo -cuyo hiperónimo se identificó en la unidad poliléxica «elemento decorativo circular»- una relativa al marco de la pintura y otra al de la escultura.

- IT-tondo ${ }^{1}$ : s.m. [Pitt.] dipinto su tela, su tavola o affrescato, di forma rotonda.

- IT-tondo ${ }^{2}$ : s.m. [Scult.] bassorilievo di forma rotonda, in marmo, ceramica, terracotta o altri materiali.

En un segundo momento, el equipo que se ocupa del español pasó a la redacción de las fichas correspondientes, consultando los corpus digitales del español, los diccionarios monolingües y bilingües, las páginas web de los museos y las traducciones de las Vidas de Vasari que forman parte de la base de datos del proyecto.

En el CDH -que fija la primera atestación de la palabra ${ }^{1}$ en 1539 con el significado de bajorrelieve- se cuentan seis ocurrencias de «tondo» en el siglo XVI y cuatro en el XVII, todas en el significado de bajorrelieve. La búsqueda en esta base de datos permite averiguar que hasta el siglo XIX la palabra se usa solo en el ámbito de la arquitectura -como sinónimo de bocel- o de la escultura.

El primer repertorio lexicográfico español donde se puede encontrar la palabra «tondo» es el Diccionario castellano con voces de ciencia y artes de Terreros (1778), que la define como voz de la arquitectura: «en la Arquitectura, bocel, ó adorno rotundo»; sin embargo, «tondo» no aparece ni en El Diccionario de las Nobles Artes de Rejón de Silva (1788) ni en el Vocabulario de términos del arte de Adeline-Mélida (1887). En 1899 «tondo» entra con la marca «arquitectura» en el diccionario académico: «(del ital. rotondo, redondo) m. Arq. Adorno circular rehundido en un paramento», definición que sigue igual hasta nuestros días. En el DRAE (1925) la Academia corrige el comentario lexicogenético, y escribe «del italiano tondo, aféresis de rotondo, y este del lat. rotundus, redondo».

Por lo que concierne a la diacronía más cercana a nuestros días, las cinco citas del CDH fechadas 1941 mencionan «tondo» como elemento arquitectónico o bajorrelieve: por ejemplo, una de ellas habla del Tondo Taddei, obra de Miguel Ángel expuesta en la Royal Academy de Londres. La primera ocurrencia de «tondo» en sentido pictórico remonta a 1988 y está entrecomillada; siempre en el CDH se puede encontrar -fechada 1996- la siguiente descripción: «En Los siete pecados capitales (Museo del Prado) se muestran cuatro círculos (tondos o huevos que simbolizan el misterio), cada uno de ellos con un novísimo». Aquí las palabras «círculo», «tondo» $\mathrm{y}$ «huevo» se utilizan de manera arbitraria como sinónimas, además porque en el cuadro de El Bosco no aparecen figuras ovaladas. En el CORPES XXI solo dos de siete ocurrencias se refieren a «tondo» como pintura; cuatro a bajorrelieves y una a la arquitectura: «También se

1Búsqueda realizada el 4/5/2017. 
añadieron motivos florales moldeados en pasta en los tondos de los remates.» (Pardo San Gil y Bartolomé 2009).

El Diccionario de términos de arte de Fatás y Borrás (2015) introduce una distinción entre «adorno circular rehundido en un paramento. En plural tondos» y «obra artística pintada o esculpida de forma redonda, semejante a un medallón. En plural tondi». En el primer caso, «tondo» se considera una palabra española perteneciente al ámbito de la arquitectura y en el segundo un extranjerismo -ya que los autores proponen el plural italiano tondi- referido al dominio de la pintura o de la escultura.

Por lo que concierne a la lexicografía bilingüe, los repertorios italiano-español asignan a la palabra tondo en su acepción artística solo un escueto equivalente, sin añadir ninguna explicación que permita comprender de qué se está hablando y sin aportar información acerca de las colocaciones. El diccionario Tam se limita a anotar: «tondo: pitt scult tondo», mientras que en el Grande Dizionario Zanichelli las dos partes del diccionario no corresponden, evidenciando de tal manera la existencia de un problema, que queda sin resolver:

- $\quad$ tondo s.m. (Archit., Pitt., Scult.) tondo, adorno circular.

- $\quad$ tondo s.m. (Arquit.) tondo.

Los Tesauros del patrimonio cultural de España identifican tres tipos de «tondo»-oscillum, escudo ducal y clípeo- y vuelven a proponer y amplía la definición del DRAE: «Motivo decorativo en relieve de forma circular, rehundido en un paramento, a veces, con molduras alrededor. Puede incluir elementos historiados».

Sin embargo, la búsqueda de «tondo» en la página web del Museo del Prado restituye once resultados de obras pintadas desde el siglo XVI al XIX; en las fichas correspondientes, la palabra puede identificar un cuadro: «Por dimensiones, formato, tema y contrastes lumínicos, este tondo se relaciona con el de la Resurrección de Campaña de la colección Várez-Fisa de Madrid» o un detalle entro de una pintura: «Ambos personajes se consideran prefiguraciones de Cristo, tema que parece ser el objeto del tríptico, como demuestran asimismo la escena de Caín y Abel situada en un tondo en el arco en ruinas [...]». La búsqueda en la página web del Museo Thyssen Bornemisza muestra un resultado análogo, la presencia de nueve obras pictóricas de los siglos XV y XVI catalogadas como «tondo»: «Tondo atribuido a Piero di Cosimo y que nos ofrece en perfecta composición un tema tradicional: La Virgen con el Niño, con un paisaje al fondo y dos ángeles que le contemplan devotamente». En una de las fichas explicativas se puede leer: «El conjunto central de esta pintura, $[. .$.$] tiene recuerdos [...] del primer Miguel Ángel, por$ la posición que adopta el Niño, que evoca la del tondo Doni de la Galleria degli Uffizi».

Tampoco las traducciones proponen soluciones coherentes: por ejemplo, en Vasari (1960) en la Vida de Botticelli tondo se traduce con las unidades pluriverbales «pintura en tondo», «cuadro en tondo» y «pintura en medallón», mientras que en Vasari (2011) se encuentra siempre «tondo $»^{2}$.

\section{Discusión y conclusiones}

Con estos supuestos, para el diccionario monolingüe del español del proyecto LBC se han elaborado tres fichas terminológicas de la palabra tondo, ejemplificadas de esta manera:

- $\quad$ ES-tondo ${ }^{1}$ : s.m. [Arq.]: ornamento redondo.

- $\quad$ ES-tondo 2 : s.m. [Esc.] bajorrelieve de forma redonda, en varios materiales.

- ES- tondo 3 : s.m. [Pint.] pintura de forma redonda sobre tabla, lienzo, o al fresco,

y relacionadas de la siguiente manera en el diccionario bilingüe:

2 Alunno (2017) presenta un estudio pormenorizado de este aspecto de la cuestión. 
- IT-tondo ${ }^{1}$ s.m. [Pint.] - ES-tondo ${ }^{3}$ s.m. [Pint.]

- $\quad$ IT-tondo $o^{2}$ s.m. [Esc.] - ES-tondo ${ }^{2}$ s.m. [Esc.]

- $\quad$ IT-tondino s.m. [Arq.] - ES-tondo ${ }^{1}$ s.m. [Arq.].

ES-tondo ${ }^{1}$ s.m. [Arq.] se relaciona con IT-tondino s.m. [Arq.], cuya ficha reza: modanatura a semicerchio usata nell'architettura classica. A su vez, tondino tiene un enlace hacia astrágalo, con el cual está relacionado por sinonimia.

El presente trabajo muestra la importancia de la creación de herramientas lexicográficas multilingües e informatizadas que puedan servir de referencia fiable a la hora de traducir un léxico complejo como el artístico, ya que se caracteriza por un elevado nivel de multidimensionalidad.

El ejemplo de las traducciones españolas de tondo pone en evidencia la falta de correspondencia biunívoca entre los términos italianos y los españoles, y la escasez de información al respecto en los diccionarios bilingües actuales más difundidos.

En efecto, en italiano tondo se utiliza en los ámbitos de la pintura y de la escultura, mientras que en español la palabra se encuentra referida a la arquitectura y escultura. La sinonimia entre «tondo» y «bocel» señalada en el Diccionario de Terreros sigue vigente hasta nuestros días, como muestra la cita del CDH procedente de Pardo San Gil y Bartolomé (2009) ${ }^{3}$. Aunque el Diccionario de Fatás y Borrás amplíe el significado de la voz al marco de la pintura, el hecho de señalar tondi como plural muestra que los autores consideran que la palabra no está aclimatada en español. No deja de maravillar que los Tesauros del Patrimonio Cultural -en los cuales se puede leer la frase «este tipo de relieves aparecen en el arte renacentista italiano, pasando posteriormente a escuelas artísticas»- no hagan referencia a «tondo» en pintura, puesto que una de las obras más conocidas del arte renacentista es precisamente el Tondo Doni de Miguel Ángel. Sin embargo, las páginas web de los museos muestran la tendencia contraria, como ejemplifican los ejemplos aportados; la falta de cursiva en el pasaje «que evoca la del tondo Doni» permite apreciar que el autor considera que la palabra está perfectamente integrada en el dominio del español, aunque modifica el nombre de la obra de Michelangelo expuesta en los Uffizi, que es «Sacra Famiglia ('Tondo Doni')».

\section{Bibliografía}

Alunno, M. (2017). Il discorso dell'arte in spagnolo e francese: dalla traduzione alla divulgazione. Uno studio a partire dalla 'Vita' di Boticelli di G. Vasari. Tesi di Laurea magistrale in Lingua, Società e Comunicazione, Università di Bologna.

Cabré M. T. (1993). La terminología: Teoría, metodología y aplicaciones. Barcelona: Editorial Antártida/Empúries.

Carpi, E. (2016). El español de la pintura y los recursos lexicográficos y terminológicos: cómo traducir al español tempera y guazzo. Cuadernos Aispi, 6, pp.143-157.

Farina, A. (2015). Un parcours du combattant: la recherche de traductions de la langue de l'art dans les ressources lexicographiques et terminologiques. L'exemple de tempera et guazzo. En E. Carpi (Ed.), Prospettive multilingue e interdisciplinari nel discorso specialistico (pp.3 3-57). Pisa: Pisa University Press.

Pardo San Gil, D. y Bartolomé García, F. R. (2009). Restauración del conjunto de la sacristía de la catedral de Santa María, Vitoria-Gasteiz. En International Institute for Conservation of

3 Sin embargo, para el Diccionario de las Nobles Artes (1788) la palabra tondino es sinónimo de bocel: «Lo mismo que Bocel: se le da este nombre quando es pequeño. F. Lor. Prim. Part. C. 29. El collarín de la coluna (toscana) es parte de ella [...] y ha de tener de alto el tondino ó bocel»; lo mismo ocurre en el Vocabulario de términos del arte de Adeline-Mélida: «Tondino [Arq.]- Voz que vale tanto como bocelillo ó baqueta (véase ésta), y que califica una moldura convexa». 
Historic and Artistic Works Grupo Español Congreso (Ed.), La restauración en el S. XXI. Función, estética e imagen. Madrid: Grupo Español del IIC.

Tarp, S. (2015). La teoría funcional en pocas palabras. Estudios de Lexicografía. Revista Mensual del Grupo de Las Dos Vidas de Las Palabras, 4, pp. 31-42.

Vasari, G. (1568). Le Vite de' più eccellenti Pittori, Scultori et Architettori. Firenze: Giunti.

Vasari, G. (1960). Vida de los más excelentes pintores, escultores y arquitectos. Estudio preliminar, traducción y notas por Julio E. Payró. Barcelona: Editorial Éxito.

Vasari, G. (2011). Las vidas de los más excelentes arquitectos, pintores y escultores italianos desde Cimabue a nuestros tiempos (antología). Estudio, selección y traducción de María Teresa Méndez Baiges y Juan María Montijano García. Madrid: Editorial Tecnos.

\section{Diccionarios y Bases de datos}

Adeline J. y Mélida J.R. (1887). Vocabulario de términos de arte. Madrid: La Ilustración Española y Americana.

Arqués, R.; Padoan, A. (2012). Il grande dizionario di spagnolo: spagnolo-italiano, italianospagnolo. Bologna: Zanichelli.

CDH: Instituto DE INVESTigación RAFAel LaPesa de la ReAl ACADEMia EsPañola (2013): Corpus del Nuevo diccionario histórico (CDH) [en linea]. < http://web.frl.es/CNDHE>

CORPES XXI: REAL ACADEMIA ESPAÑOLA: Banco de datos (CORPES XXI) [en línea]. Corpus del Español del Siglo XXI (CORPES). <http://www.rae.es>

DRAE (1899): Real Academia Española (1899). Diccionario de la Lengua Castellana. Madrid: Imprenta de los Sres. Hernando y Compañía.

DRAE (1925): Real Academia Española (1925). Diccionario de la Lengua Española. Madrid: Calpe.

Fatás G., y Borrás G.M., (2015). Diccionario de términos de arte y elementos de arqueología, heráldica y numismática. Madrid: Alianza Editorial.

Rejón de Silva, D.A. (1788). Diccionario de las Nobles Artes. Segovia: Imprenta de Antonio Espinosa.

Rico L. y Martínez C. (Dirs.) (2003). Diccionario Técnico Akal de Conservación y Restauración de Bienes Culturales. Madrid: Ediciones Akal.

Tam, L. (2009). Grande Dizionario Hoepli. Spagnolo. Milano: Hoepli.

Terreros y Pando, E. de (1788). Diccionario castellano con las voces de ciencias y artes [...]. Madrid: Viuda de Ibarra.

\section{Páginas web}

Museo del Prado: https://www.museodelprado.es/coleccion/obras-de-arte?searchObras=tondo Museo Thyssen-Bornemisza: https://www.museothyssen.org/buscador?key=tondo Galleria degli Uffizi: http://www.polomuseale.firenze.it/inv1890/scheda.asp
Tesauros
del
Patrimonio
Cultural
de
España:

http://tesauros.mecd.es/tesauros/bienesculturales/1016477.html 